\title{
Collation and Research of Health Keeping Thoughts and Methods in Dunhuang Posthumous Writing
}

\author{
Xue $\operatorname{Lin}^{1, a}$ \\ ${ }^{1}$ Economic and Trade College, Gansu University of Chinese Medicine, Lanzhou, 730000, China \\ ${ }^{\mathrm{a}}$ email
}

Keywords: Health Keeping, Food therapy, Dunhuang Posthumous Writing

\begin{abstract}
There are rich records of health keeping in the Dunhuang Posthumous Writing, and most of them are combined with diagnosis and treatment. At that time, these thoughts and methods played an important role in health and conditioning. This paper analyzes the thoughts and methods of food intake and sexual love in Dunhuang Posthumous Writing, which not only plays a certain role in the excavation and collation of medical literature in Dunhuang, but also has a reference value for the study of modern medicine.
\end{abstract}

\section{Introduction}

At the beginning of twentieth century, Dunhuang cave was discovered, and the importance of the Dunhuang Canon in hundreds of volumes of papers. They are an important treasure of medieval culture China the astrological research. The content of Dunhuang Posthumous Writing can be divided into two parts: religious books and secular books. Religious books accounted for $80 \%$, including the law, theory, interpretation, eulogy, Tuo Ronnie, oration, China. "Diamond Sutra", "Lotus Sutra" like many copies, also found that some Chinese books have been lost, such as the Sui and Tang Dynasties repeatedly banned three orders of teaching of Scripture, as well as a number of apocryphal. In addition to the Buddhist scriptures, religious texts also find several other religious texts. Among them, there are more thanTaoist papers. The north part of which is long of the code, with compensation and compiling work, enrich the literature treasure house of our country; the other part is the existing literature handed down, but in order to complement the work of collation, provides a rare version. Found in the Mogao Grottoes in Dunhuang during the Sui and Tang Dynasties document, traditional Chinese medicine has a considerable amount of data, including medical volume more than 30, carrying more than 1000 first party. These doctors except for a few scattered before the Sui and Tang Dynasties handed books, mostly before the local folk medicine and single prescription manuscript. These lost by the discovery, to promote the study of Manichaeism and Nestorianism that scholars. In the medical literature has long lost books and doctors. There are also many ancient books and documents written by ancient ethnic minorities and western regions in the Dunhuang Posthumous Writing, which play an important role in the study of the history of the western regions, languages and the history of transportation between China and the west. In short, Dunhuang manuscripts with its extensive content, precious and famous, to promote the study of medieval Chinese and Central Asian history, archaeology, linguistics, text fields science, ethnology, religion, literature, art, Shu Zhixue, historical geography and history of science and technology. Many countries in the world have corresponding research institutions and organizations engaged in research work of Dunhuang manuscripts, and the formation of the twentieth century international discipline.

\section{Thoughts and Methods of Food Therapy in Dunhuang Posthumous Writing}

Balanced Diet. Dunhuang manuscripts "auxiliary verse to" drug law viscera explicitly "five dishes for the charge, five for help, for raising crops, five animals for the benefit of nutrition collocation principle, this view and law theory "contained in the" grain for the support, five for help, five livestock, five dishes for the charge, while the clothes of the odor together to benefit Qi "point of 
view line, conforming to dietary nutrition: the principle of diversification of the modern" five food "," five tobacco "," grain "," five animals "are not only the basic human diet and food sources, namely grain cereals, fruits and vegetables, animal food, more important is that dietary structure should be complete, the proportion of appropriate and sufficient quantity, to avoid the partial eclipse, so as to ensure the carbohydrate, protein, dietary fiber, vitamins, and other inorganic elements Balanced intake of nutrients. Human diet nutrition practice shows that this concept of diet is conducive to the maintenance of health, prevention and treatment of diseases, in the history of world dietary science, this is the earliest balanced dietary records, so far, modern nutrition cannot go beyond. Balanced diet refers to the amount of energy and nutrients in the diet is adequate, complete range, the proportion of appropriate; dietary nutrients and the needs of the body to maintain balance between. The structure of diet should be reasonable, not only to meet the physiological needs of the body, but also to avoid the imbalance of dietary composition and excessive nutrients, resulting in unnecessary burden on the body and metabolic disorder in the near future or in the long run. The human body needs more than 42 kinds of nutrients, including various types of protein, fats, carbohydrates, vitamins, minerals, essential trace elements and water.

Medicine Food Homology. Many foods are both food and medicine, and food and drugs can also prevent disease. In primitive society, people find all kinds of food and taste and efficacy of drugs in the process of looking for food, recognizing that many foods can be medicine, many drugs can also eat, are very difficult to strictly distinguish between. Work, strain; loss, deficiency, so the term refers to all kinds of internal strain deficiency lesions, organs dysfunction, loss of vitality, consistent with modern medicine, sub-health, health is a state between physiology and pathology, is often the first one viscera Qi and blood, Yin, Yang's loss, and involved him dirty. We should pay more attention to reinforcing the day after tomorrow, diet is closed: the Dunhuang manuscripts "auxiliary verse to" pay special attention to medication of viscera organs, reinforcing and reducing mutual possession, following the "five dishes for the charge five, fruit, grain to raise livestock, five put forward five strain these dietotherapy prescription diet prescriptions for the benefit", "Q - organ law inheritance theory" understanding of the human organs according to law, and the choice of food, food and medicine to the side, Use the same food, shows the conditioning principle: from Dunhuang manuscripts "auxiliary verse to" load "viscera medication inspection with gist of various drugs for the purpose of understanding, can by" and "this figure is possible to effect", the food, food and medicine with methods of treatment with strain may it is early for prescription method, to make up for the shortcomings of Zhang Zhongjing's prescription. This is the basis of the theory of "medicine and food being alike" and the basis of food therapy. The concept of traditional Chinese medicine is that all the animals and plants, minerals and so on belong to the category of traditional Chinese medicine, and Chinese medicine is a very big concept of medicine. Therefore, strictly speaking, in Chinese medicine, drugs and food are not divided, is relative: drugs are also food, and food is also a drug; the side effects of food are small, while the side effects of drugs are large.

Food Therapy in Treating Illness. There are a large number of diet formulas in Dunhuang's Posthumous Writing, which are various in ingredients and flexible in form, which reflect the essence of TCM syndrome differentiation and treatment, and pay attention to the significance of different dietary forms in different diseases. In the selection of ingredients, special attention to the western regions of specialty, cattle and sheep products and pungent, warm, fragrant, scattered products. The two matches will not only increase the edible value, and increase the warm tonic, fill the essence of therapeutic value. Cattle and sheep are in the ancient western regions is one of the main products, local food, the first 20 components of compound prescription clear diet statistics in Dunhuang show that the Posthumous Writing, prescription application of cattle products were 11 first. In the dietary form the most western characteristic is "Bo Ren" this ancient form of food used in diet prescriptions are rarely seen in the past, this diet ancient literature: Bo Ren first appeared in the northern and Southern Dynasties, the law and the patch phase. all emergency fire cooked by boiling. "China diet dictionary" has more detailed explanation to it: "with gravy cooling good taste after mixing the flour, knead a thick strip, cut into two inches long, in the water soaked with boiling and then put the noodles while rubbing thin fire fall cooked: white light and lovely. there is no doubt that the ancient and this 
was one of the similar patches, pasta northern important is still very popular in Northwest china. Food is also divided into reinforcing and reducing elements. Supplementary food can enhance people's resistance, increase strength, suitable for weak physique edible, and empirical anti constitution patients caused by constipation, sweat out, virus accumulation in vivo, hypertension, inflammation, poisoning and other adverse symptoms of diarrhea; food can help will be eliminated from the body of the virus, improve constipation, positive physical congestion, inflammation and other symptoms.

\section{Thoughts and Methods of Sexual Health in Dunhuang Posthumous Writing}

Treatment of Male Diseases. No matter what stage to the development of human civilization, people pursuit of sex will not stop, and constantly looking for sex method can make sex both maximize the satisfaction of health, this is not only the individual mental and physical needs, is to breed, reproductive health and improve the quality of life is of great significance. Treat male make generous ("praying Party Book" "Cistanche, oysters, Dendrobium two. And balm painted on the penis, that is, a large test". For the external use of Yin Yang Fang plants for the fleshy stems of Cistanche or many. It is sweet, sour, salty and warm. It has the function of invigorating kidney and benefiting essence, moistening dryness and smoothing intestines. It can be used to treat men impotence, female infertility. The "dictionary of Chinese medicine" cited Materia Medical "refers to the treatment of male female in the vast Yin Yang, do not produce." Oyster salty, astringing Yin Yang, Dendrobium or its various plant stems. It is sweet, light and salty, and has the function of strengthening Yin and strengthening yang. Treat male to large and square "in her book" party ". Bai Siren five points, four points, seven horse penis surgery, Guangxi heart two, aconite two, bulk liquor service, big spoon, again on the service times." In order to invigorate the spleen, invigorate the heart and invigorate the Yang, it is necessary to promote the strengthening of the heart, invigorate the mind, calm the nerves, invigorate the heart, aconite, the penis, warm the kidney and invigorate the kidney, and take the wine as the guide and increase the effect of promoting blood circulation to invigorate the arteries and invigorate the liver. Treatment.

Treatment of Female Diseases. In the white line of yin and Yang "sex music Fu" is a representative of Dunhuang medical works, reflects the concept of open. Dunhuang harmony fully embodies the importance of both men and women of happiness, not only cure male Yin Xiao Fang, and there are a lot of treatment of women with cold, Yin cold wide, generous side of "a woman happy, strong man" also reflects the importance of women. The ancient coins symbol of reproductive function, later will be tied to the women's folk coins in the neck, "money", to produce children hanging clothes wrapped coins buried underground to sterilization, so the coins here has the double meaning of praying and contraception; Dunhuang folk, the dog is a multiparous animal, white the dog milk in woman vagina when sexual intercourse, has a treatment effect for free and long married infertile women; mulberry hole because like the female vagina, so that the hole in the mulberry grass has extraordinary reproductive capacity, the burning ash, water will have to kimono. In the middle, deep and long shook his head." A woman is happy, a man is a good man. Three things for the end of treatment, sexual dysfunction of men and women, men and women to stimulate the function of women to achieve a happy, strong man good purpose. Although the party in Shandra to taste bittersweet, but five of the acid, although warm, but warm but not dry, lung, heart and kidney by three, acid convergence, notifying the lung, who willing to be, heart, kidney failure, energy dissipation, or Yin two injuries, are available. Polygala bitter pungent and warm, bitter relief Wen Tong Xin effect, can reduce the firelight, kidney due to heart, and the heart and kidney function of traffic. Szechuan hard and warm, into the kidney, warming kidney yang, bitter and dampness, for the treatment of impotence disease to medicine with cold herbs palace. The Dunhuang medical home to three divided into the end of a female, sub channel, so deep, but his head for a long time.

Treatment of Childlessness Problems. Seven powders and four takes three pills, often such as "Fu Xing Jue" in the medication of viscera Nourishing Kidney Decoction can cure male idiopathic infertility. Her treatment of infertility treatment including woman without children, girl, do not swallow, no male children, treatment of white dog milk, men and women, no woman will Takako, 
mulberry hole grass, female man, one party rule, and make the correct number of loss. The ancient thought sex not only enjoy happiness. Dunhuang hospital also reflected the people eager in her mood, boys are particularly prominent in Dunhuang folk expression of the Tang dynasty. From the woman and the man praying all female has evident. Parents are eager to give birth to boys, adding labor for the family, but also a matter of procreation. "And" one big social origin is clearly patriarchal. Many of the children, to the modern view of absurd, but reflects the folk custom. "No mortal, when there are five couples work seven losses, caused by diseases of the virtual lean"; with the same period of Chao Yuanfang also believes that "kidney main bone marrow and essence, asthenia kidney weakness, so also less semen". Chinese medicine has a long history of diagnosis and treatment of male infertility, and many ancient doctors have had a brilliant exposition. In the ancient doctors stressed the role of internal in male infertility, the incidence of disease, mainly due to changes in the trial for the exploration of the internal organs of the body, in exploring the pathogenesis is deficiency to advocates, Xie Sheng is second, which caused by the deficiency of infertility accounted for a considerable proportion. Therefore, ancient doctors believe that deficiency syndrome is the main type of male infertility, especially kidney deficiency.

\section{Conclusion}

Chinese medicine is broad and profound. Its exquisite theory and technology are all embodied in the medical and pharmaceutical use to a certain extent. The thoughts and methods of health preserving in Dunhuang Posthumous Writing are worth using for reference and popularization. Some valuable prescriptions and drugs in Dunhuang Posthumous Writing can be developed and utilized through further analysis and research.

\section{Acknowledgement}

The paper is the result of Opening Foundation Project of Co-constructing Key Labs by Province and Ministry of Dunhuang Medicine and Transformation from 2015 to 2016 named Collation and Research of Health Keeping Thoughts and Methods in Dunhuang Posthumous Writing (Grant No. DHYX1516-03) led by Xue Lin.

\section{References}

[1] Liang Yonglin, Li Jintian, Liu Jia, Li Yingcun. Shushu thought research of Dabuxietang in Fu Xing Jue of Dunhuang post famous book [J]. China Journal of Traditional Chinese Medicine and Pharmacy, 2012, 27(2): 277-279.

[2] Wang Bo, Li Danlin, Liu Xiping. Studies on the nursing content of medication in Dunhuang book relics [J]. Global Traditional Chinese Medicine, 2014, 7(9): 694-696.

[3] Sun Shouhua, On Medical Recipes of Sexual Harmony in DunHuang YiShu [J]. Western Journal of Traditional Chinese Medicine, 2012, 25(3): 44-48.

[4] Xing Jiaming, Yan Xingke, Zhao Zhongting, Liu Anguo, Han Yadi, Research and Application of Moxibustion Therapy in Dunhuang Manuscripts [J]. Chinese Journal of Information on TCM, 2016, 23(8): 132-136. 